\title{
Effect of DHU001, a Polyherbal Formula, on Dinitrofluorobenzene-induced Contact Dermatitis (Type I allergy)
}

\author{
Hyeung-Sik Lee', Byung-Chang Lee ${ }^{2}$ and Sae-Kwang $\mathrm{Ku}^{2}$ \\ ${ }^{1}$ Department of Clinical Laboratory Science, College of Health and Therapy, \\ ${ }^{2}$ Development Anatomy and Histology, College of Oriental Medicine, Daegu Haany University, Gyeongsan 712-715, Korea
}

(Received February 16, 2010; Revised May 7, 2010; Accepted May 10, 2010)

\begin{abstract}
The effect of DHU001, a mixed herbal formula consisted of 7 types aqueous extracts for various respiratory disorders were evaluated on 2,4-dinitrofluorobenzene (DNFB)-induced contact dermatitis, type I allergic model. Contact dermatitis was induced by sensitization with dinitrophenyl-derivatized ovalbumin (DNP-OVA) and DNFB challenge as antigen. Two different dosages of DHU001 (300 and $150 \mathrm{mg} / \mathrm{kg}$ ) were orally administered to DNP-OVA sensitization mice once a day for 7 days with reference material, dexamethasone $(15 \mathrm{mg} / \mathrm{kg}$, intraperitoneal treatment). End of 7 days oral administration of DHU001 extracts or intraperitoneal treatment of dexamethasone, the changes on the edematous changes and scratching behavior were measured. Immediate after DNFB challenge on ear or paw of DNP-OVA sensitized mice, increases of ear and paw thicknesses and weights were detected with anterior ear skin (dermis to epidermis) thickness and paw scratching behavior increases. However, these contact dermatitis signs induced by DNFB treatment were reduced by treatment of the both different dosages of DHU001 and dexamethasone, respectively. The results obtained in this study suggest that oral treatment of DHU001 extracts also has relatively favorable effects on contact dermatitis.
\end{abstract}

Key words: DHU001, Polyherbal Formula, 2,4-Dinitrofluorobenzene, Mouse, Contact Dermatitis

\section{INTRODUCTION}

Atopic dermatitis is a chronic, itchy skin condition that is very common in children but may occur at any age. It is also known as eczema and atopic eczema. It is the most common form of dermatitis. Atopic dermatitis usually occurs in people who have an 'atopic tendency'. This means they may develop any or all of three closely linked conditions atopic dermatitis, asthma and hay fever (allergic rhinitis). Often these conditions run within families with a parent, child or sibling also affected. A family history of asthma, eczema or hay fever is particularly useful in diagnosing atopic dermatitis in infants. Atopic dermatitis affects 15 20\% of children but only 1 2\% of adults (Kiczuk et al., 2004; Foroughi et al., 2005; Nakahara and Furue, 2005; Williams, 2005). In cases of allergic disease with chronic and severe pruritus, such as atopic dermatitis, contact dermatitis and urticaria, are accompanied by severe pruritus (Lorette and Vaillant, 1990; Klecz and Schwartz, 1992). Therefore, it is

Correspondence to: Sae-Kwang Ku, Department of Anatomy and Histology, College of Oriental Medicine, Daegu Haany University, 290, Yugok-dong, Gyeongsan-si, Gyeongsangbuk-do 712-715, Korea E-mail: gucci200@hanmail.net very important not only to treat the allergy but also to inhibit scratching of the lesion (Ishiguro et al., 2002).

2,4-Dinitrofluorobenzene (DNFB) is used as an intermediate in the synthesis of pesticides (algicides) and some pharmaceuticals such as flurbiprofen. It is also used as a sensitizing agent and hapten in laboratory immunology and as a reagent to identify the terminal amino acids in a protein chain (Wang et al., 1998; Perez et al., 2004). The repeated application of DNFB to the ears of mice results in a typical allergic dermatitis and the simultaneous production of IgE antibody against DNFB (Nagai et al., 1997ab). Repeated application of DNFB onto the mouse ear causes the ear to swell accompanied by the rise of serum specific IgE levels (Nagai et al., 1997b). Thickening of epidermis, formation of scabs, and infiltration of abundant inflammatory cells are also induced (Ueda et al., 2003). Therefore DNFB-induced contact dermatitis model is a useful for evaluation of a potential treatment candidate for type I allergic dermatitis (Matsuda et al., 2002). The effects of a drug would be based on the ear swelling and incidence of scratching behaviors in this model (Matsuda et al., 2002).

Topical steroids have been a popular choice for treating various cutaneous disorders; however, the potential for significant local and systemic adverse events, like skin atrophy 
and hypothalamic-pituitary-adrenal (HPA) axis suppression, has limited their use (Gupta and Chow, 2004). Among glucocorticoids, dexamethasone is the one of widely used topical steroids on atopic dermatitis (Ganir et al., 1996; Matsumoto et al., 2005) and has been used as a reference drug on development of the new anti-allergic agents (Shichinohe et al., 1996; Ohtsuka et al., 2003).

DHU001 is a mixed herbal formula consisted of 7 types aqueous extracts - Ficis fructus, Liriopis tuber, Platycodi radix, Schisandrae fructus, Glycyrrhizae radix, Zingiberis rhizome and Menthae herba and being developed for various respiratory disorders. Among 7 types of herbal components of DHU001, Liriopis tuber (Park and Geon, 2003), Platycodi radix (Kim et al., 2004), Schisandrae fructus (Narimanian et al., 2005; Rhyu et al., 2006), Glycyrrhizae radix (Sun and Pan, 2006), Zingiberis rhizome (Aimbire et al., 2007; Ghayur et al., 2008) and Menthae herba (Shin, 2003) have been used for treating various respiratory symptoms. In addition, antiinflammatory effects of Platycodi radix (Ahn et al., 2005), Glycyrrhizae radix (Park et al., 2005), Zingiberis rhizome (Aimbire et al., 2007; Minghetti et al., 2007) have been reported, and DHU001 itself also showed anti-inflammatory effects on the mouse xylene-induced acute inflammation (Back et al., 2008). Therefore, it also considered that oral administration of DHU001 will be ameliorated the atopic dermatitis because the allergic dermatitis induced by DNFB also showed quite similar phenomena like general inflammatory responses (Nagai et al., 1997a, b; Ueda et al., 2003). When the surface of skin is inflamed, cracked or raw, many of these sting or burn when first applied. This irritation will lessen as the atopic dermatitis improves related to antiinflammation activity (Skinner, 2004; Rossetti et al., 2005).

In the present study, the effects of DHU001 aqueous extracts were evaluated on the DNFB-induced contact dermatitis, and the results were compared with dexamethasone, $15 \mathrm{mg} / \mathrm{kg}$ intraperitoneally treated group.

\section{MATERIALS AND METHODS}

Experimental animals. Eighty hundred male ICR mice (6-wk old upon receipt, SLC, JAPAN) were used after acclimatization for 10 days. Animals were allocated five per polycarbonate cage in a temperature $\left(20 \sim 25^{\circ} \mathrm{C}\right)$ and humidity (40 45\%) controlled room. Light : dark cycle was $12 \mathrm{hr}$ : $12 \mathrm{hr}$ and feed (Samyang, Korea) and water were supplied free to access. About half animals are selected based on preliminary test (over 10\% increases of ear thickness) and each of 5 mice per group was used for ear edema test and paw scratching test.

Preparation of DHU001. The herbal compositions of DHU001 were listed in Table 1. Each herbal component was purchased from Cho-Heung Pharmaceutical Ind. Co. (Daegu, Korea) after confirmation of the morphology under microscopy. Approximated amounts of each herbal component was mixed (317.5 g) and boiled in $2 l$ of distilled water for 2 hours and then filtrated. The filtrate was decompressed using a rotary vacuum evaporator (Lab. Camp, Daejeon, Korea) and lyophilized in a programmable freezedryer (IlShin Lab, Daejeon, Korea). Total acquired lyophilized extracts were $34.93 \mathrm{~g}$ (yield 11\%). Powders of extracts were stored in a desiccator to protect against light and moisture. It was well dissolved up to $100 \mathrm{mg} / \mathrm{m} l$ concentration levels and appeared to be a deep brown solution.

Administration of drugs. Two different dosages of DHU001 aqueous extracts (300 and $150 \mathrm{mg} / \mathrm{kg}$; dissolved in distilled water) were orally administered to Dinitrophenylderivatized ovalbumin (DNP-OVA) sensitization mice once a day for 7 days, and $15 \mathrm{mg} / \mathrm{kg}$ of dexamethasone-water soluble (Sigma, MO, USA; dissolved in saline) was intraperitoneal administered as same frequencies as DHU001 extracts. In DNFB control, distilled water was orally administered instead of DHU001 extracts as same methods.

DNFB-induced contact dermatitis. DNP-OVA was prepared according to the method described by Eisen and Belmam (1953) and was used as an antigen. DNP-OVA were prepared by mixing $200 \mathrm{mg}$ OVA (Sigma, MO, USA) with $100 \mathrm{mg}$ 2,4-dinitrofluorobenzene (Sigma, MO, USA) for $18 \mathrm{~h}$ at room temperature. The number of DNP groups coupled to OVA was 3.2/molecule.

Table 1. Herbal composition of DHU001 used in this study

\begin{tabular}{|c|c|c|}
\hline Herbs & Scientific name & Amounts (g) \\
\hline Ficis fructus & Ficus carica Linn. & 140 \\
\hline Liriopis tuber & Liriope spicata Lour. & 45 \\
\hline Platycodi radix & Platycodon grandiflorum Jacq. & 60 \\
\hline Schisandrae fructus & Schisandra chinensis Baill & 22.5 \\
\hline Glycyrrhizae radix & Glycyrrhiza uralensis Fisch & 15 \\
\hline Zingiberis rhizoma recens & Zingiber officinale Roscoe & 15 \\
\hline Menthae Herba & Mentha arvensis Linne var piperascens & 20 \\
\hline Total & 7 types & 317.5 \\
\hline
\end{tabular}

All herbs were purchase from Cho-Heung Pharmaceutical Ind. Co. (Daegu, Korea) and yield 11\% aqueous extracts were acquired. 
Animal selection: Mice were sensitized by an intraperitoneal injection of a mixture of DNP-OVA $(10 \mu \mathrm{g})$ and aluminum hydroxide gel $(1 \mathrm{mg})$ in saline $(0.2 \mathrm{~m} l)$. After one week, the mice were challenged by painting $10 \mu l$ of $0.1 \%$ DNFB solution in ethanol on the inside and outside of the right and left ears as previously (Watanabe et al., 1999). About half animals showing an increment of percent change (over $15 \%$ ) of ear swelling were chosen.

Sensitization: Next day of animal selection, the mice were again sensitized by the mixture of DNP-OVA $(10 \mathrm{mg})$ and aluminum hydroxide gel $(1 \mathrm{mg})$ in saline $(0.2 \mathrm{~m} l)$.

Antigen challenge: Challenged by painting $10 \mu l$ of $0.1 \%$ DNFB solution in ethanol on the inside of the right ears for ear swelling test, or on the hind paws of each mouse for scratching test.

Ear and Paw thickness measurements. The thicknesses of the intact and induced ear and/or paw were measured using an electronic digital caliper (Mytutoyo, Japan) $1 \mathrm{hr}$ after the DNFB challenge. The \% increment of thickness was expressed in percentage difference between the thickness of intact and induced sides and calculated as [(thicknesses of induced sides-thicknesses of intact sides)/ thicknesses of intact sides] $\times 100$.

Ear and Paw weight measurements. $1 \mathrm{hr}$ after the DNFB challenge, circular sections of both sides of ear and paw were taken using a cork borer with a 7-mm diameter as previously described (Lee and $\mathrm{Ku}, 2008$ ), and weighed using an automatic electronic balance. The $\%$ increment of weights was expressed in percentage difference between the weights of intact and induced sides and calculated as [(weights of induced sides - weights of intact sides)/weights of intact sides] $\times 100$.

Scratching behavior detection. From immediately after DNFB challenges on the paw, the incidences of scratching behavior on the whole body and the site challenged with DNFB were counted for $1 \mathrm{hr}$. Behavior observation was done as previously method (Matsuda et al., 2002). In brief, after at least $1 \mathrm{hr}$ min acclimation to the experimental environment, mouse behaviors were monitored small windows served for counting the scratching. The scratching behavior was defined as movement of the hind limb excluding the movement for gait.

Histopathology. After measuring of thickness or weights, DNFB challenged ear was sampled and fixed in 10\% neutral buffered formalin after cross trimming. After paraffin embedding, $3 \sim 4 \mu \mathrm{m}$ sections were prepared. Representative sections were stained with hematoxylin and eosin (H\&E) for light microscopical examination. After that the histological profiles of individual ear were evaluated.

Histomorphometry: The thicknesses of anterior skin parts - from epidermis to dermis (ear cartilages were excluded) were measured using automated CCD image analyzer (DMI300 Image Processing; DMI, Korea) under magnification 100 of microscopy (Nikkon, Japan) at body regions of ear.

Statistical analyses. Multiple comparison tests for different dose groups were conducted. Variance homogeneity was examined using the Levene test. If the Levene test indicated no significant deviations from variance homogeneity, the obtain data were analyzed by one way ANOVA test followed by least-significant differences multi-comparison (LSD) test to determine which pairs of group comparison were significantly different. In case of significant deviations from variance homogeneity were observed at Levene test, a nonparametric comparison test, Kruskal-Wallis $\mathrm{H}$ test was conducted. When a significant difference is detected in the Kruskal-Wallis H test, the Mann-Whitney U (MW) test was conducted to determine the specific pairs of group comparison, which are significantly different. Statistical analyses were conducted using SPSS for Windows (Release 6.1.3., SPSS Inc., USA) and a $P$ value $<0.05$ was considered significant.

\section{RESULTS}

Changes on the ear and paw thicknesses. As results of ear and paw thickness measurements to observe the

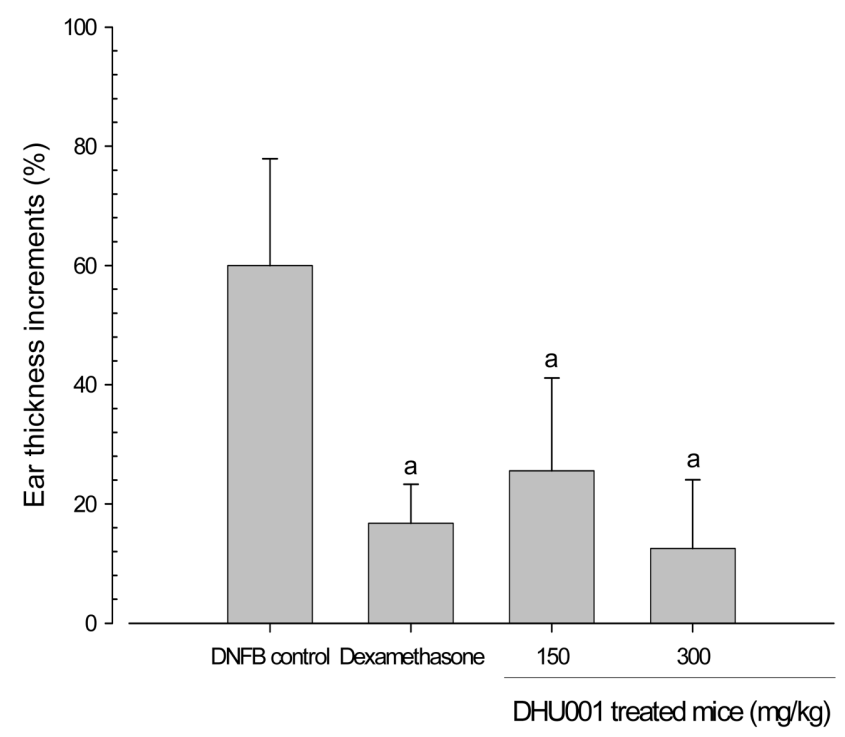

Fig. 1. Changes of ear thickness increments detected after 7 days of continuous oral treatment of DHU001 in DNFB-sensitized mice. Significant decreases of ear thickness increments were detected in dexamethasone and two different dosages of DHU001 aqueous extracts treated groups as compared with DNFB control, and DHU001 aqueous extracts showed clear dose-dependent inhibitions on the ear thickness increments in this study. Values are expressed mean \pm S.D. of five mice; ${ }^{a} p<$ 0.01 as compared with DNFB control by LSD test. 


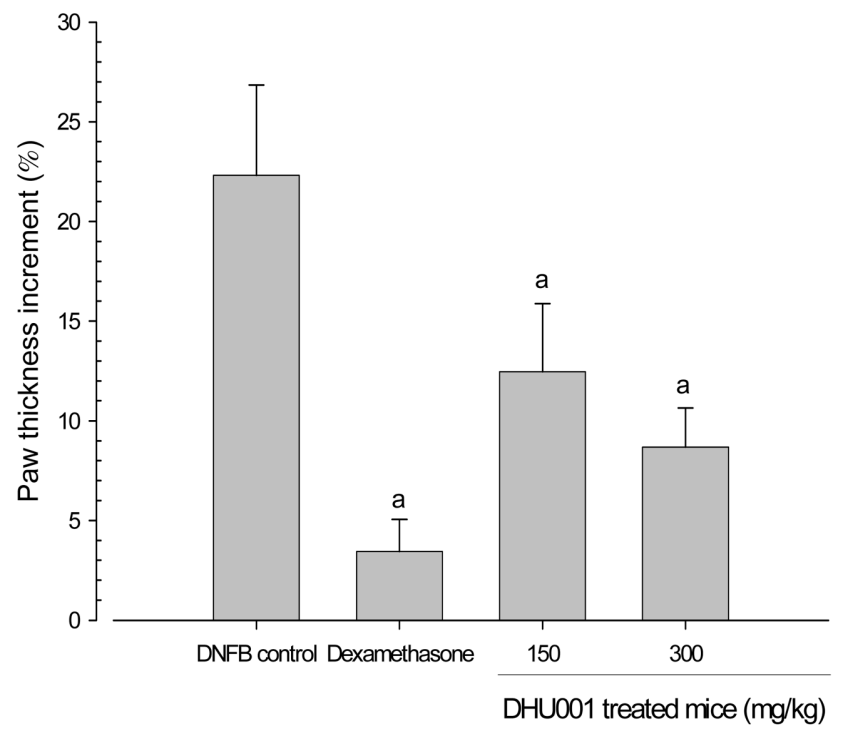

Fig. 2. Changes of paw thickness increments detected after 7 days of continuous oral treatment of DHU001 in DNFB-sensitized mice. Significant decreases of paw thickness increments were detected in dexamethasone and two different dosages of DHU001 aqueous extracts treated groups as compared with DNFB control, and DHU001 aqueous extracts showed clear dose-dependent inhibitions on the paw thickness increments in this study. Values are expressed mean \pm S.D. of five mice; ${ }^{a} p<$ 0.01 as compared with DNFB control by LSD test.

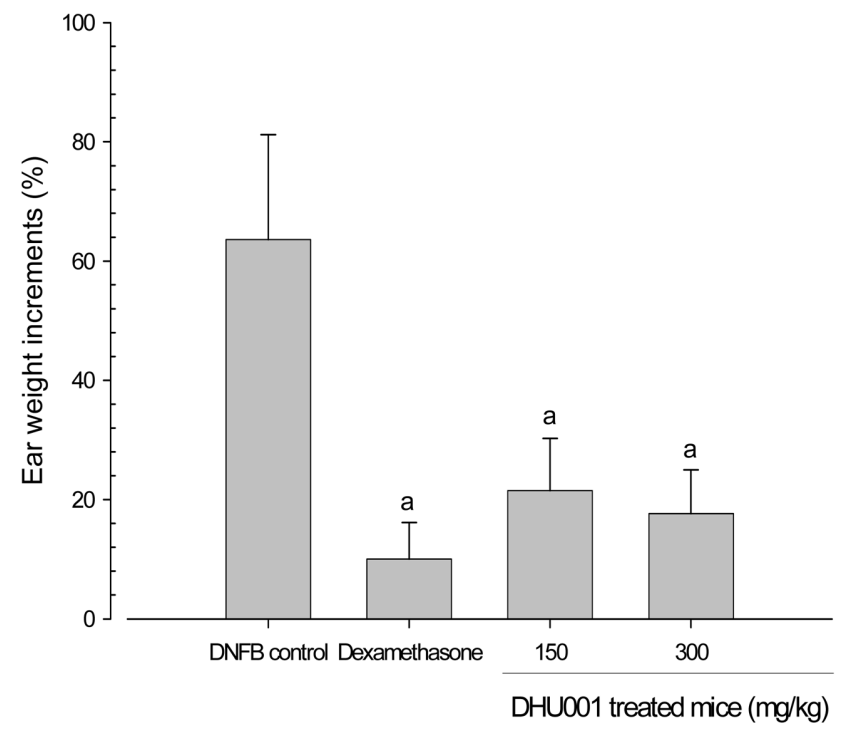

Fig. 3. Changes of ear weight increments detected after 7 days of continuous oral treatment of DHU001 in DNFB-sensitized mice. Significant decreases of ear weight increments were detected in dexamethasone and two different dosages of DHU001 aqueous extracts treated groups as compared with DNFB control, and DHU001 aqueous extracts showed clear dose-dependent inhibitions on the ear weight increments in this study. Values are expressed mean \pm S.D. of five mice; ${ }^{a} p<0.01$ as compared with DNFB control by LSD test.

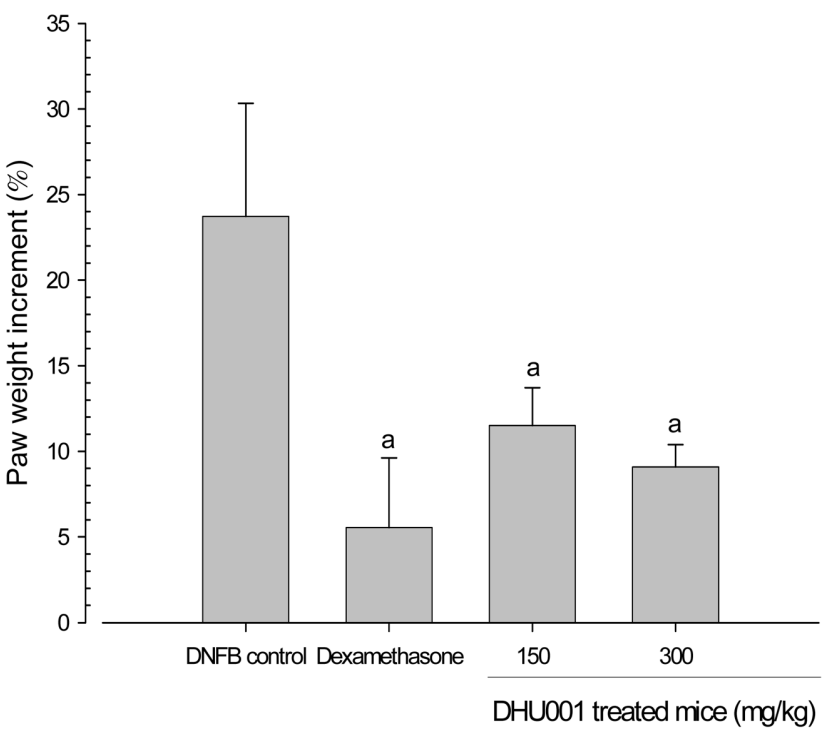

Fig. 4. Changes of paw weight increments detected after 7 days of continuous oral treatment of DHU001 in DNFB-sensitized mice. Significant decreases of paw weight increments were detected in dexamethasone and two different dosages of DHU001 aqueous extracts treated groups as compared with DNFB control, and DHU001 aqueous extracts showed clear dose-dependent inhibitions on the paw weight increments in this study. Values are expressed mean \pm S.D. of five mice; ${ }^{a} p<$ 0.01 as compared with DNFB control by LSD test.

edematous changes, dramatical decreases of ear and paw thickness increments were detected in all tested groups as compared with DNFB control, respectively (Fig. 1 and 2).

Changes on the ear and paw weights. To detect the inducement of edematous changes, the ear and paw weights were measured on individual mice in this study. As shown in Fig. 3 and 4 , significant $(P<0.01)$ decreases of ear and paw weight increments were detected in all tested groups as compared with DNFB control, respectively.

Changes on the scratching numbers. The effects of DHU001 extracts on the pruritis were observed by measuring the frequency of scratching behaviors. In the present study, significant $(P<0.01)$ decreases of scratching behaviors were detected in all tested groups as compared with DNFB control (Fig. 5).

Histopathological changes. Marked increases of skin thickness were detected in DNFB control with severe dermis related edematous changes - loosening of connective tissues at histopathological observations, but these histopathological changes were markedly inhibited by treatment of dexamethasone, DHU001 extracts 300 and $150 \mathrm{mg} / \mathrm{kg}$ treated groups, respectively (Fig. 6). The increases of skin thickness as detected by histomorphometry were detected in 


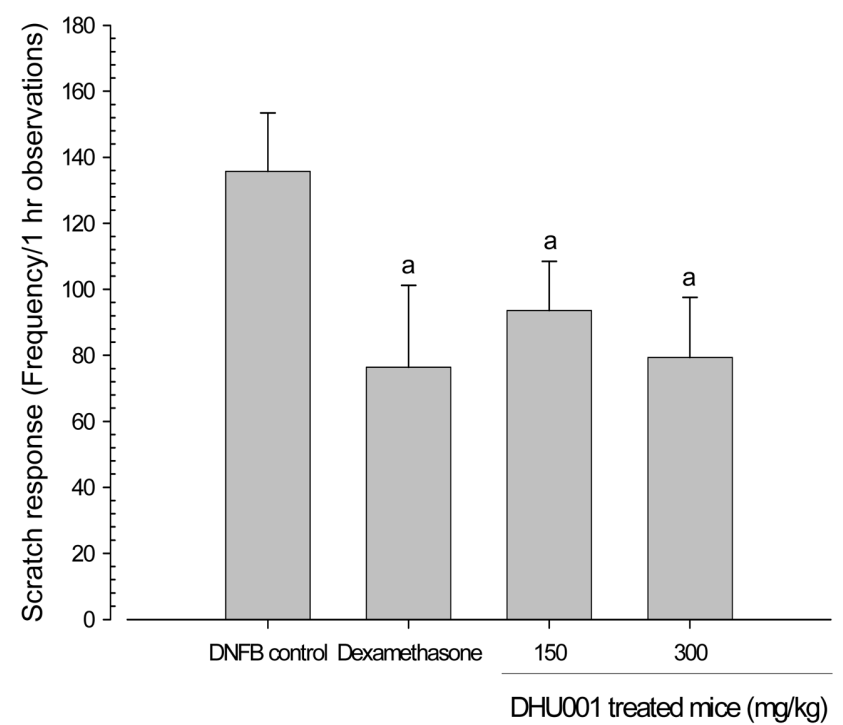

Fig. 5. Changes of scratch responses detected after 7 days of continuous oral treatment of DHU001 in DNFB-sensitized mice. Significant decreases of scratch responses were detected in dexamethasone and two different dosages of DHU001 aqueous extracts treated groups as compared with DNFB control, and DHU001 aqueous extracts showed clear dose-dependent inhibitions on the scratch responses in this study. Values are expressed mean \pm S.D. of five mice; ${ }^{a} p<0.01$ as compared with DNFB control by LSD test.

DNFB control due to severe dermis edematous changes, but these edematous changes were markedly inhibited by treatment of dexamethasone and both different dosages of DHU001 extracts (Fig. 7).

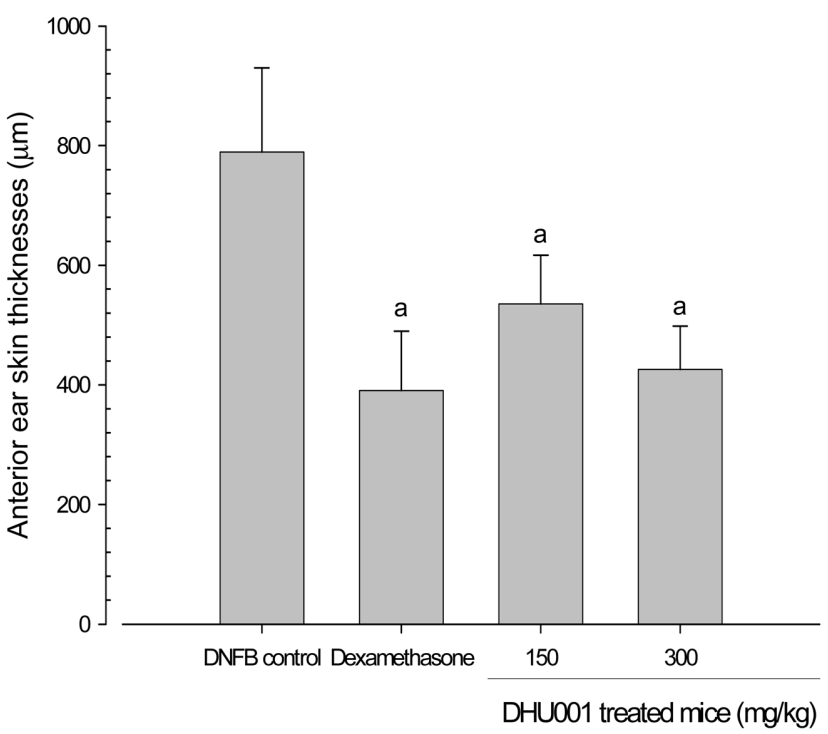

Fig. 7. Changes of anterior ear skin thicknesses detected after 7 days of continuous oral treatment of DHU001 in DNFB-sensitized mice. Significant decreases of anterior skin thicknesses were detected in dexamethasone and two different dosages of DHU001 aqueous extracts treated groups as compared with DNFB control, and DHU001 aqueous extracts showed clear dose-dependent inhibitions on the anterior skin thicknesses increments in this study. Values are expressed mean \pm S.D. of five mice; ${ }^{a} p<0.01$ as compared with DNFB control by LSD test.

\section{DISCUSSION}

Because the allergic dermatitis evoked by DNFB also showed quite similar phenomena like general inflammatory
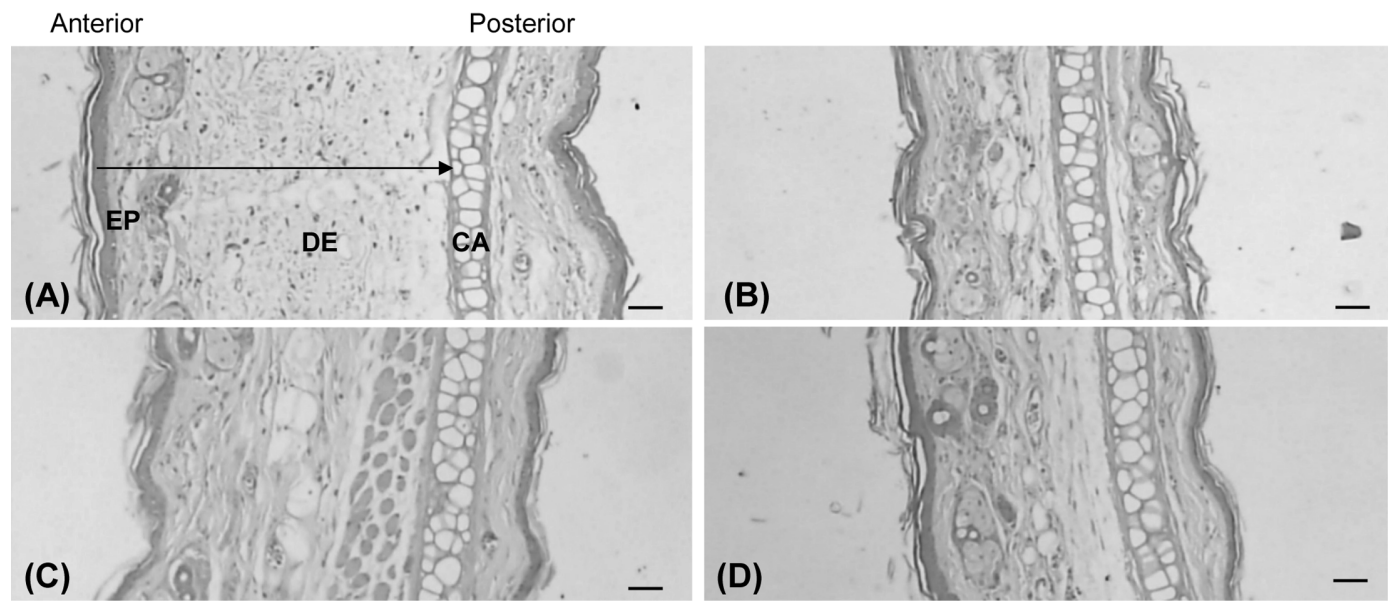

Fig. 6. Histopathological observations of the ear in the DNFB control (A), dexamethasone (B), DHU001 150 (C) and 300 (D) mg/kg treated groups. Note that marked increases of skin thickness (arrow) were detected in DNFB control with severe dermis edematous changes, but these increases were markedly inhibited by treatment of dexamethasone and two different dosages of DHU001 aqueous extracts treated groups as compared with DNFB control, and DHU001 aqueous extracts showed clear dose-dependent inhibitions on the ear histopathological changes induced by DNFB treatment in the present study. CA, ear hyaline cartilages; EP, epidermis; DE, dermis; All H\&E stain; Scale bars $=80 \mu \mathrm{m}$. 
responses (Nagai et al., 1997a, b; Ueda et al., 2003), and DHU001 itself, also showed anti-inflammatory effects (Back et al., 2008) with anti-inflammatory effects of (Ahn et al., 2005), Glycyrrhizae radix (Park et al., 2005), Zingiberis rhizome (Aimbire et al., 2007; Minghetti et al., 2007) among 7 types of herbal components of DHU001. Therefore, we considered that oral administration of DHU-001 will be ameliorated the atopic dermatitis. In the present study, contact dermatitis was induced by sensitization with DNP-OVA and DNFB challenge as antigen. Two different concentrations of DHU001 aqueous extracts (300 and 150 $\mathrm{mg} / \mathrm{kg}$ ) were orally administered to DNP-OVA sensitization mice once a day for 7 days with dexamethasone as reference. End of administration of DHU001 extracts and dexamethasone, the changes on the edematous changes and scratching (pruritis) behavior were measured.

Immediate after DNFB challenge on DNP-OVA sensitized mice ear and paw, increases of ear and paw thickness and weights were detected with increases of anterior ear skin (dermis to epidermis) thickness and paw scratching behaviors. However, these edematous and allergic changes results from DNFB treatment were dramatically inhibited by treatment of the both two different dosages of DHU001 extracts and dexamethasone in the present study.

Repeated application of DNFB onto the mouse ear causes swell accompanied by the rise of serum specific IgE levels (Nagai et al., 1997b). Thickening of epidermis, formation of scabs, and infiltration of abundant inflammatory cells are also induced (Ueda et al., 2003). Therefore DNFB-induced contact dermatitis model has been used for evaluation of a potential treatment candidate for type I allergic dermatitis (Matsuda et al., 2002). DNFB to mice sensitized by DNPOVA caused edemas similar to an occurrence of atopic dermatitis is $\operatorname{IgE}$ antibody dependent appearing $1 \mathrm{hr}$ after application of DNFB (Watanabe et al., 1999). In the present study, marked edematous changes, increment of thickness and weights were detected on DNFB challenged ear and paw with pruritis. However, these edematous changes induced by DNFB challenges were markedly inhibited by administration of the both dosages of DHU001. It is considered as direct evidences that DHU001 can be ameliorated the type I allergic dermatitis including atopic dermatitis and these are considered as result of anti-inflammatory effects of DHU001, itself, already reported (Back et al., 2008).

The favorable effects of DHU001 on the DNFB-induced contact dermatitis detected in the present study are considered to relate with the anti-inflammatory effect of DHU001 mediated immunomodulatory effects of the herbal components already reported (Yoon et al., 2003; Tan and Vanitha, 2004; Sun and Pan, 2006), because immunomodulatory agents can reduce inflammation (Ramprasath et al., 2006) and, furthermore, NO synthase inhibitors can reverse several classic inflammatory symptoms (Amin et al., 1995). Because oxidative stress are also involved in the pathogene- sis of various types dermatitis (Wright et al., 2005; Geronikaki and Gavalas, 2006) and antioxidants have been ameliorated the DNFB-induced contact dermatitis (Steenvoorden and Beijersbergen van Henegouwen, 1999; Gordon et al., 2008), the antioxidant effects of DHU001 were considered as one of key mechanisms, at least on the ameliorate effects of DHU001 on the DNFB-induced contact dermatitis detected in this study. Antioxidative effects of all 7 herbal components of DHU001; Ficis fructus (Pèrez et al., 2003), Liriopis tuber (Han et al., 1981), Platycodi radix (Wang et al., 2004), Schisandrae fructus (Ko and Lam, 2002), Glycyrrhizae radix (Zhou and Xu, 1992), Zingiberis rhizome (Masuda et al., 2004) and Menthae herba (Baliga et al., 2003); were already demonstrated.

The results obtained in this study suggest that oral treatment of DHU001 also has relatively favorable effects on allergic dermatitis mediated by immunomodulatory and antioxidant effects related anti-inflammatory effects of DHU001 itself, or herbal components.

\section{ACKNOWLEDGMENT}

This research was supported by a grant from Daegu Haany University Ky-rin Fund, 2009 (2009-901-43).

\section{REFERENCES}

Ahn, K.S., Noh, E.J., Zhao, H.L., Jung, S.H., Kang, S.S. and Kim, Y.S. (2005). Inhibition of inducible nitric oxide synthase and cyclooxygenase II by Platycodon grandiflorum saponins via suppression of nuclear factor-kappaB activation in RAW 264.7 cells. Life Sci., 76, 2315-2328.

Aimbire, F., Penna, S.C., Rodrigues, M., Rodrigues, K.C., LopesMartins, R.A. and Sertié, J.A. (2007). Effect of hydroalcoholic extract of Zingiber officinalis rhizomes on LPS-induced rat airway hyperreactivity and lung inflammation. Prostaglandins Leukot Essent Fatty Acids, 77, 129-138.

Amin, A.R., Vyas, P., Attur, M., Leszczynska-Piziak, J., Patel, I.R., Weissmann, G. and Abramson, S.B. (1995). The mode of action of aspirin-like drugs: effect on inducible nitric oxide synthase. Proc. Natl. Acad. Sci. USA, 92, 7926-7930.

Back, Y.D., Lee, H.S. and Ku, S.K. (2008). Effects of DHU001, a mixed herbal formula on acute inflammation in mice. Toxicol. Res., 24, 189-194.

Baliga, M.S., Jagetia, G.C., Rao, S.K. and Babu, K. (2003). Evaluation of nitric oxide scavenging activity of certain spices in vitro: a preliminary study. Nahrung, 47, 261-264.

Eisen, H.N. and Belmam, S. (1953). Studies of hypersensitivity to low molecular weight substances. II. Reactions of some allergenic substituted dinitrobenzenes with cysteine or cystine of skin proteins. J. Exp. Med., 98, 533-549.

Foroughi, S., Thyagarajan, A. and Stone, K.D. (2005). Advances in pediatric asthma and atopic dermatitis. Curr. Opin. Pediatr., 17, 658-663.

Ganir, E.M., Capulong, M.C., Tahara, K., Akasawa, A. and Iikura, Y. (1996). Treatment of atopic dermatitis in children: the impor- 
tance of skin care and environmental control. Acta Paediatr. Jpn., 38, 702-704.

Geronikaki, A.A. and Gavalas, A.M. (2006). Antioxidants and inflammatory disease: synthetic and natural antioxidants with anti-inflammatory activity. Comb. Chem. High Throughput Screen, 9, 425-442.

Ghayur, M.N., Gilani, A.H. and Janssen, L.J. (2008). Ginger attenuates acetylcholine-induced contraction and $\mathrm{Ca} 2+$ signalling in murine airway smooth muscle cells. Can. J. Physiol. Pharmacol., 86, 264-271.

Gordon, J.S., Wolanin, P.M., Gonzalez, A.V., Fela, D.A., Sarngadharan, G., Rouzard, K., Perez, E., Stock, J.B. and Stock, M.B. (2008). Topical N-acetyl-S-farnesyl-L-cysteine inhibits mouse skin inflammation, and unlike dexamethasone, its effects are restricted to the application site. J. Invest. Dermatol., 128, 643-654.

Gupta, A.K. and Chow, M. (2004). Prednicarbate (Dermatop): profile of a corticosteroid. J. Cutan. Med. Surg., 8, 244-247.

Han, B.H., Yoo, S.Y., Park, M.H. and Lee, H.J. (1981). Antioxidant activity screening on crude drugs. J. Pharmacogn., 12, 66.

Ishiguro, K., Oku, H., Suitani, A. and Yamamoto, Y. (2002). Effects of conjugated linoleic acid on anaphylaxis and allergic pruritus. Biol. Pharm. Bull., 25, 1655-1657.

Kiczuk, K., Chodorowska, G. and Krasowska, D. (2004). The care of the child with atopic dermatitis. Ann. Univ. Mariae Curie Sklodowska [Med.], 59, 503-507.

Kim, S.S., Lee, S.C., Shin, H.D., Shin, M.K., Kim, J.H. and Song, H.J. (2004). Studies on allergy asthma effect of Radix Platicodi. Kor. J. Herbology, 19, 61-70.

Klecz, R.J. and Schwartz, R.A. (1992). Pruritus. Am Family Physician, 45, 2681-2686.

Ko, K.M. and Lam, B.Y. (2002). Schisandrin B protects against tert-butylhydroperoxide induced cerebral toxicity by enhancing glutathione antioxidant status in mouse brain. Mol. Cell Biochem., 238, 181-186.

Lorette, G. and Vaillant, L. (1990). Pruritus: current concepts in pathogenesis and treatment. Drugs, 39, 218-223.

Masuda, Y., Kikuzaki, H., Hisamoto, M. and Nakatani, N. (2004). Antioxidant properties of gingerol related compounds from ginger. Biofactors, 21, 293-296.

Matsuda, H., Tomohiro, N., Ido, Y. and Kubo, M. (2002). Antiallergic effects of cnidii monnieri fructus (dried fruits of Cnidium monnieri) and its major component, osthol. Biol. Pharm. Bull., 25, 809-812.

Matsumoto, K., Mizukoshi, K., Oyobikawa, M., Ohshima, H., Sakai, Y. and Tagami, H. (2005). Objective evaluation of the efficacy of daily topical applications of cosmetics bases using the hairless mouse model of atopic dermatitis. Skin Res. Technol., 11, 209-217.

Minghetti, P., Sosa, S., Cilurzo, F., Casiraghi, A., Alberti, E., Tubaro, A., Loggia, R.D. and Montanari, L. (2007). Evaluation of the topical anti-inflammatory activity of ginger dry extracts from solutions and plasters. Planta Med., 73, 1525-1530.

Nagai, H., Hiyama, H., Matsuo, A., Ueda, Y., Inagaki, N. and Kawada, K. (1997a). FK-506 and cyclosporin A potentiate the IgE antibody production by contact sensitization with hapten in mice. J. Pharmacol. Exp. Ther., 283, 321-327.

Nagai, H., Matsuo, A., Hiyama, H., Inagaki, N. and Kawada, K. (1997b). Immunoglobulin $\mathrm{E}$ production in mice by means of contact sensitization with a simple chemical, hapten. J. Allergy Clin. Immunol., 100, S39-44.

Nakahara, T. and Furue, M. (2005). Mechanism of pathogenesis in atopic dermatitis. Nippon Rinsho, 63, 80-85.

Narimanian, M., Badalyan, M., Panosyan, V., Gabrielyan, E., Panossian, A., Wikman, G. and Wagner, H. (2005). Impact of Chisan (ADAPT-232) on the quality-of-life and its efficacy as an adjuvant in the treatment of acute non-specific pneumonia. Phytomedicine, 12, 723-729.

Ohtsuka, E., Kawai, S., Nojima, H., Andoh, T., Kamimura, K. and Kuraishi, Y. (2003). Inhibitory effect of azelastine on allergic itch-associated response in mice sensitized with mosquito salivary glands extract. J. Pharmacol. Sci., 91, 263-266.

Park, E., Kum, S., Wang, C., Park, S.Y., Kim, B.S. and SchullerLevis, G. (2005). Anti-inflammatory activity of herbal medicines: inhibition of nitric oxide production and tumor necrosis factor-alpha secretion in an activated macrophage-like cell line. Am. J. Chin. Med., 33, 415-424.

Park, J.H. and Geon, D.G. (2003). Pharmacognostical studies on the Chinese crude drug "Maig Moon Dong". Kor. J. Pharmacogn., 34, 6-9.

Perez, A., Narayan, S. and Sansom, J. (2004). Occupational contact dermatitis from 2,4-dinitrofluorobenzene. Contact Dermatitis, 51, 314.

Pèrez, C., Canal, J.R. and Torres, M.D. (2003). Experimental diabetes treated with Ficus carica extract: effect on oxidative stress parameters. Acta Diabetol., 40, 3-8.

Ramprasath, V.R., Shanthi, P. and Sachdanandam, P. (2006). Immunomodulatory and anti-inflammatory effects of Semecarpus anacardium LINN. Nut milk extract in experimental inflammatory conditions. Biol. Pharm. Bull., 29, 693-700.

Rhyu, M.R., Kim, E.Y., Yoon, B.K., Lee, Y.J. and Chen, S.N. (2006). Aqueous extract of Schizandra chinensis fruit causes endothelium-dependent and -independent relaxation of isolated rat thoracic aorta. Phytomedicine, 13, 651-657.

Rossetti, G., Laffitte, E., Eigenmann, P.A., Lubbe, J., Hohl, D. and Hofer, M.F. (2005). Treatment of atopic dermatitis: practical approach. Rev. Med. Suisse, 1, 501-504.

Shichinohe, K., Shimizu, M. and Kurokawa, K. (1996). Effect of M-711 on experimental skin reactions induced by chemical mediators in rats. J. Vet. Med. Sci., 58, 419-423.

Shin, T.Y. (2003). Inhibition of immunologic and nonimmunologic stimulation-mediated anaphylactic reactions by the aqueous extract of Mentha arvensis. Immunopharmacol. Immunotoxicol., 25, 273-283.

Skinner, R. (2004). Role of topical therapies in the management of cutaneous disease. J. Cutan. Med. Surg., 8, 22-31.

Steenvoorden, D.P. and Beijersbergen van Henegouwen, G. (1999). Protection against UV-induced systemic immunosuppression in mice by a single topical application of the antioxidant vitamins C and E. Int. J. Radiat. Biol., 75, 747-755.

Sun, H.X. and Pan, H.J. (2006). Immunological adjuvant effect of Glycyrrhiza uralensis saponins on the immune responses to ovalbumin in mice. Vaccine, 24, 1914-1920.

Tan, B.K. and Vanitha, J. (2004). Immunomodulatory and antimicrobial effects of some traditional Chinese medicinal herbs: a review. Curr. Med. Chem., 11, 1423-1430.

Wang, C., Schuller Levis, G.B., Lee, E.B., Levis, W.R., Lee, D.W., Kim, B.S., Park, S.Y. and Park, E. (2004). Platycodin D and D3 
isolated from the root of Platycodon grandiflorum modulate the production of nitric oxide and secretion of TNF-alpha in activated RAW 264.7 cells. Int. Immunopharmacol., 4, 1039-1049.

Wang, H.W., Tedla, N., Lloyd, A.R., Wakefield, D. and McNeil, P.H. (1998). Mast cell activation and migration to lymph nodes during induction of an immune response in mice. J. Clin. Invest., 102, 1617-1626.

Watanabe, C., Satoh, T., Tahara, E., Murakami, K., Hayashi, K., Hase, K., Andoh, T., Kuraishi, Y., Kadota, S., Nagai, H. and Saiki, I. (1999). Inhibitory mechanisms of glycoprotein fraction derived from Miscanthus sinensis for the immediate phase response of an IgE-mediated cutaneous reaction. Biol. Pharm. Bull., 22, 26-30.

Williams, H.C. (2005). Clinical practice. Atopic dermatitis. $N$. Engl. J. Med., 352, 2314-2324.
Wright, R.J., Cohen, R.T. and Cohen, S. (2005). The impact of stress on the development and expression of atopy. Curr. Opin. Allergy Clin. Immunol., 5, 23-29.

Ueda, Y., Sone, T., Inagaki, N. and Nagai, H. (2003). Effects of prednisolone on the cutaneous reaction and skin barrier function in mice treated with a hapten. Biol. Pharm. Bull., 26, 618621.

Yoon, Y.D., Han, S.B., Kang, J.S., Lee, C.W., Park, S.K., Lee, H.S., Kang, J.S. and Kim, H.M. (2003). Toll-like receptor 4dependent activation of macrophages by polysaccharide isolated from the radix of Platycodon grandiflorum. Int. Immunopharmacol., 3, 1873-1882.

Zhou, Y. and Xu, R. (1992). Antioxidative effect of Chinese drugs. Zhongguo Zhong Yao Za Zhi, 17, 368-369, 373. 